\title{
Study of the geometry in a 3D flow-focusing device
}

\author{
Elena Castro-Hernández ${ }^{1}$ Maarten P. Kok ${ }^{2} \cdot$ Michel Versluis $^{2} \cdot$ \\ David Fernandez Rivas ${ }^{3}$
}

Received: 7 July 2015 / Accepted: 10 January 2016

(C) Springer-Verlag Berlin Heidelberg 2016

\begin{abstract}
We present a numerical and experimental study on a non-planar three-dimensional design of a microfluidic flow-focusing device for the well-controlled generation of monodisperse micron-sized droplets. Three relevant geometric parameters were identified: the distance between the inner inlet channel and the outlet channel, the width of the outlet channel, and its length. Simulation data extracted from a full parameter study and finite element simulations yielded four optimum designs that were then fabricated using soft lithography techniques. Under the predicted operating conditions, micro-droplets of a size of $\sim 1 \mu \mathrm{m}$ in diameter are obtained from a channel $50 \mu \mathrm{m}$ in width. This work represents an important breakthrough in the practical use of flow-focusing devices delivering a ratio of constriction to droplet size of 50 times, with the advantage of reduced clogging of the micro-channel, greatly improving the control and reliability of the device.
\end{abstract}

Keywords Flow-focusing · Microfluidics · Jet · Micro-droplets

Elena Castro-Hernández

elenacastro@us.es

Michel Versluis

m.versluis@utwente.nl

1 Área de Mecánica de Fluidos, Departamento de Ingeniería Aeroespacial y Mecánica de Fluidos, Universidad de Sevilla, Avenida de los Descubrimientos s/n, 41092 Seville, Spain

2 Physics of Fluids Group, MESA+ Institute of Nanotechnology, University of Twente, P.O. Box 217, 7500 AE Enschede, The Netherlands

3 Mesoscale Chemical Systems, MESA+ Institute of Nanotechnology, University of Twente, P.O. Box 217, 7500 AE Enschede, The Netherlands

\section{Introduction}

In many practical applications, such as in the food industry, fine chemicals, and pharmaceutical production, a reduction in the droplet size in emulsions is desired, but it is limited by the conventional emulsification techniques. Currently, rotor-stator homogenizers used for this purpose produce large emulsion droplets in the order of $100 \mu \mathrm{m}$. Developing equipment with dimensions larger than available for research purposes is an ongoing challenge the industry faces (Schroën et al. 2015). For example, the complexities in producing monodisperse emulsions and nanosized droplets with low energy requirements in cross-flow membrane emulsification are due to the morphology of the membrane (wetting properties, pore size, porosity, and pore shape) and defined by the desired droplet sizes and applied pressure (Gijsbertsen-Abrahamse 2004). The ultimate goal for industrial-scale emulsification is the control on a single geometrical parameter that could then be used to generate a range of droplets size with a single device. Microfluidic devices are a potential solution since their use has been demonstrated in the production of homogeneous monomer droplets and emulsions, for chemistry and biology applications with low energy input, and high throughputs in the order of $L / h$ (Nisisako and Torii 2008; Sugiura et al. 2001; Engl et al. 2008).

There are two extensively used types of devices for the production of droplets in microfluidics : (1) co-flow devices where two immiscible fluids flow in parallel (Utada et al. 2007; Marín et al. 2009; Castro-Hernández et al. 2009) and (2) flow-focusing devices in which both streams flow through a constriction with a length of the same order as its width (Gañán-Calvo 1998; Anna et al. 2003; Cubaud and Mason 2008; Garstecki et al. 2005; Lee et al. 2009). In cross-flow emulsification, uniform droplets of 2-10 times, 
the pore size can be formed; in spontaneous emulsification droplets of 3-6 times, the height of the terrace/plateau can be achieved (Schroën et al. 2015). In both methods, it is mandatory that the surfaces remain wetted by the continuous phase, even when emulsifiers, such as proteins, are dissolved to avoid loss of emulsification.

Despite the differences of both configurations, two global regimes can be identified: (1) a dripping regime, where drops are formed right at the tip of the injection tube (axisymmetric case) or at the entrance of the outlet channel (2D case), and (2) a jetting regime characterized by the generation of an extended jet that eventually breaks up due to capillary instability. These designs can be implemented in axisymmetric geometries, by means of concentric capillary tubes, or in $2 \mathrm{D}$ configurations using soft lithography techniques.

In practice, an axisymmetric geometry using glass capillary-based devices, as was shown by Utada et al. (2005), requires a perfect alignment between the tubes by matching the outer diameter of a cylindrical capillary tube to the inner dimension of a square one. Since the outer fluid needs to be pumped through the small space between the four corners, its viscosity is limited to below $100 \mathrm{cP}$. Gañán-Calvo (1998) reported the production of monodisperse droplets using axisymmetric flow-focusing devices. Although the outer viscosity was not limited, the alignment between the injection tube and the constriction (a plate with a small orifice) was a tedious task, which limited efficient control of the device.

Micro-fabricated devices in a 2D geometry have been used extensively since the early ages of microfluidics. Particularly, hydrodynamic focusing has been implemented for cytometry, particle counting, and fluorescence-activated cell sorting (Simonnet and Groisman 2005; Sim et al. 2010; Chiu et al. 2013) and other applications that exploit the laminar flow conditions and swirl effects inside microchannels. Attempts to focus the flow at the axis of microchannels utilized different strategies with some limitations, such as undesired entrapment of air bubbles, vorticity, local turbulence, boundary separation in the flow, and the notorious clogging of the micro-channels during operation. Most solutions to these problems have complicated operational conditions. Micro-channel designs made of different materials further complicate the fabrication process lead to undesired wetting properties and additional laboratory equipment to operate introducing a considerable footprint (Scott et al. 2008; Golden et al. 2009; Rosenauer et al. 2011; Zhuang et al. 2012; Frankowski et al. 2013; Dhanaliwala et al. 2012).

Silicon and glass are more difficult and more expensive to micro-machine than the popular casting and curing of poly-dimethyl siloxane (PDMS) over silicon and SU-8 molds, where the cured PDMS slab is bonded to a glass slide, or other materials to form the micro-channel. However, the use of different materials for the different walls of a micro-channel poses a disadvantage, due to intrinsic differences in the wetting properties of the materials used. Attempts at simplifying hydrodynamic focusing in $2 \mathrm{D}$ and 3D microfluidic devices were possible using novel techniques such as femto-laser micromachining (Paiè et al. 2014), with the drawback of using more expensive materials like fused silica, troublesome chemicals (hydrogen fluoride), and laser operation, making it a costly technology.

The aim of this work is to study the influence of the relevant parameters in a 3D non-planar flow-focusing device for the production of micron-sized droplets $(1 \mu \mathrm{m}$ in diameter) generated in the jetting regime inside a channel of $50 \mu \mathrm{m}$ in width. The main advantage of using a truly 3D device made of a single material is obtaining an axisymmetric jet centered at the outlet channel, and as the jet breaks up into droplets, there is no contact with the walls of the channel and hence no influence of the wetting properties of the material. This then excludes the need of chemicals to regulate the hydrophobicity of the microchannels, and there is no effect of changing wetting properties of the channel wall at extended operation times. The latter represents an attractive feature for a multitude of applications, especially in micro-emulsification, where materials with limited machining possibilities are commonly used (Schroën et al. 2015). Specifically, stainless steel is preferable compared to glass, silicon, or polymers such as PDMS, but such a device is technically challenging to fabricate using conventional clean-room or microfabrication techniques.

\section{Materials and methods}

\subsection{Mask fabrication}

The mold used to cast two PDMS slabs that make the channel was prepared in a similar way as described in the literature (Anderson et al. 2000; Carlier et al. 2004). Three different levels of SU-8 were spun on a silicon wafer to obtain the final thicknesses $H_{1}=50 \mu \mathrm{m}, H_{2}=100 \mu \mathrm{m}$, $H_{3}=25 \mu \mathrm{m}$ (see Table 1). The first level was achieved with SU-8 25 (MicroChem Corp.), at 1885 rpm, whereas the other two with SU-8 50 at $2000 \mathrm{rpm}$. This difference in channel height is the key design characteristic to obtain the focusing effect (see Fig. 1).

The design proposed in this study is composed of an inlet channel for the dispersed phase with height $H_{1}$ and width $W_{1}$, one inlet channel for the continuous phase with height $\mathrm{H}_{2}$ and width $W_{2}$, that splits around and intersects with the dispersed phase channel at a 90 degree angle, and the emulsion outlet channel with a height $\mathrm{H}_{3}$ and width $W_{3}$, as shown in Fig. 1. 
(a)

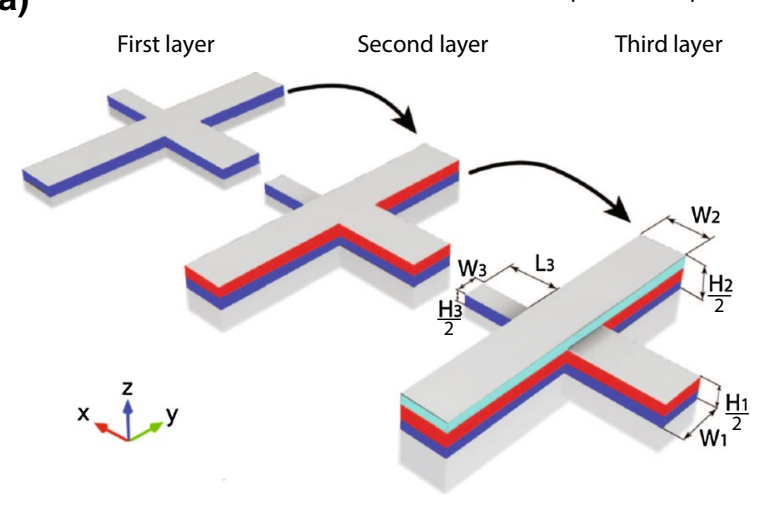

(b)

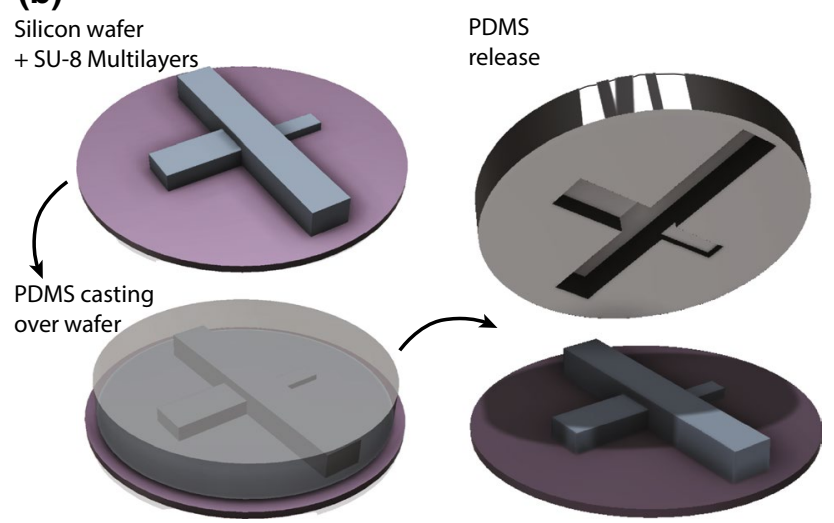

Fig. 1 Schematic figure showing a the main dimensions of the channel intersection in a 3D flow-focusing device, and $\mathbf{b}$ the simplified 3D channel mold and the PDMS lift-off process. The heights of the different layers in the mold are half the dimensions of the actual device and numerical model

PDMS (Polydimethylsiloxane, Sylgard 184, Dow Corning) mixed at a 10:1 curing ratio was placed into the silicon wafers and cured at a temperature of $75^{\circ} \mathrm{C}$ during $1 \mathrm{~h}$. After peeling off and punching the holes for the tubing connections in one of the replicas, two pieces of the same PDMS design were treated in a oxygen plasma cleaner (Harrick plasma, PDC-002) at maximum power for $4 \mathrm{~min}$. The alignment between the two parts was made by hand using an inverted microscope (Zeiss, Axiovert 40C) with a $10 \times$ objective. The resulting device was baked in the oven at a temperature of $75{ }^{\circ} \mathrm{C}$ for $1 \mathrm{~h}$ to increase the bonding strength (see Fig. 1).

\subsection{Experimental setup}

Silicon oil with a viscosity $\mu_{o}=1000 \mathrm{cP}$ (Sigma-Aldrich) was used as outer fluid, whereas ultrapure water (MilliQ, Millipore Corporation, Billerica, MA, USA) was used as inner fluid. The surface tension, $\sigma$, between oil and water was lowered from 40 to $4 \mathrm{mN} / \mathrm{m}$ by adding $60 \mathrm{mM}$ of sodium dodecyl sulfate (SDS, Sigma-Aldrich) to the water.
In order for the outer velocity to be sufficiently high and to ensure accurate control of the inner flow rate, both streams were supplied from pressurized vessels controlled by two high-precision air pressure regulators (Norgren, 11-818100) and two digital manometers (Digitron 2000P). Additionally, a large flow resistance along the inner line was given by a peek tubing with $127 \mu \mathrm{m}$ inner diameter. The setup was placed under an inverted microscope (Zeiss, Axiovert 40C) which was connected to either a high-speed camera (Photron, SA-X2 type 1080K-M4) with a resolution of $1024 \times 1024 \mathrm{px}^{2}$ and a field of view of $775 \times 775 \mu \mathrm{m}^{2}$ when operated at an acquisition rate of $2 \times 10^{4} \mathrm{fps}$. Similarly, a high-resolution CCD camera (Lumenera Lm165M) can be mounted to the microscope with a resolution of $800 \times 800 \mathrm{px}^{2}$ and a field of view of $5160 \times 5160 \mu \mathrm{m}^{2}$, corresponding to $6.45 \mu \mathrm{m} /$ pixel.

\subsection{Numerical methods}

Boundary element method simulations were used before (Castro-Hernández et al. 2012) to establish solutions for the flow in an axisymmetric co-flow device. Briefly, the Stokes equation for both inner (i) and outer (o) streams, $-\nabla P_{i, o}+\mu_{i, o} \nabla^{2} U_{i, o}=0$ is solved, where the inner flow has a parabolic velocity profile with a curvature inversely proportional to the inner-to-outer viscosity ratio, $\mu_{i} / \mu_{o}$ (capital letters indicate a dimensional quantity). Here, $U_{i}$ and $U_{o}$ are the mean velocities of the inner and outer streams, respectively, and $C a_{o}=\mu_{o} U_{o} / \sigma$ is the outer capillary number. Moreover, the axial velocity at the jet interface does not significantly depends on $\mu_{i} / \mu_{o}$; hence, the velocity at the jet interface and the pressure gradient are given, in a first approximation, by the outer stream. Based on the slender jet geometry and classical Taylor approximation (Taylor 1964), the original problem could be studied as the sum of two simpler ones: (1) the outer flow without the presence of the inner one, and (2) a perturbation induced by the inner stream which can be modeled as a source distribution located at the axis, with an initially unknown amplitude. The axial component of the outer velocity is $u_{o}=u_{x}+f r^{2}$, where $u_{x}$ is the velocity at the axis $(r=0)$ and $f=\partial^{2} u / \partial r^{2}(r=0)$. These functions are independent of the three dimensionless parameters controlling the droplet generation process for the co-flow configuration $\left(\mu_{i} / \mu_{o}\right.$, $U_{i} / U_{o}$ and $C a_{o}$ ), and are characteristic for each geometry. For a non-axisymmetric flow focusing, these assumptions are also valid.

In a 3D non-planar flow-focusing device, the asymptotic values of the flow velocity in the $\mathrm{x}$-direction, $u_{x}$, and the orthogonal gradient in the shear rate in the center of the channel, $f=\partial^{2} u_{x} / \partial y^{2}$, should be reached as close to the junction as possible. A large magnitude of $f$ has a favorable effect on the production of small droplets. 
To investigate the influence of several of the design parameters involved in the geometry, specifically the distance between the inner inlet channel and the outlet channel, $W_{2}$, and the width of the outlet channel, $W_{3}$, finite element simulations were performed using the COMSOL Multiphysics package (COMSOL, Inc., MA, USA).

The simulations encompass the geometry as shown in Fig. 1. Lengths and widths used are the same as shown in Table 1, with $L_{3}$ kept constant at $250 \mu \mathrm{m}$. A no-slip boundary conditions applies to all the walls, with defined flow rate for the three inlet channels, and an atmospheric pressure outflow condition at the outlet. Since the pressures inside the experimental device are unknown, flow rate boundary conditions are chosen rather than pressure boundary conditions. The center inlet provides $0.1 \mu \mathrm{L} / \mathrm{min}$, while both side entrances combined provide $10 \mu \mathrm{L} / \mathrm{min}$. The code solves the stationary incompressible Navier-Stokes equations on the domain. The highest Reynolds number reached is of order 10, guaranteeing steady, laminar flow.

\section{Results and discussion}

\subsection{Inlet channel width}

Figure 2 shows the streamlines calculated for a 3D flowfocusing device for two different distances between the inlet channel and the outlet channel $W_{2}: 25$ and $50 \mu \mathrm{m}$. It displays the confinement of the inner fluid along the symmetry plane, $z=0$. It also shows a larger recirculation cell for the device with the smaller $W_{2}$.

Figure 3 depicts the downstream evolution of $u_{x}$, nondimensionalized by its maximum, $U_{x, \max }$, for the two different geometries.

It shows that a smaller $W_{2}$ leads to a minor increase in the gradient $\partial u_{x} / \partial x$, but that a smaller $W_{2}$ shifts the location of the maximum gradient more upstream. For an increased width of the outlet channel, $W_{3}$, it is shown that the maximum value of the gradient decreases.

Figure 4 shows the downstream evolution of the orthogonal shear rate gradient $f$, non-dimensionalized using the velocity $U_{x, \max }$, and the outlet channel width $W_{3}$, for the different geometries studied. A decrease in $W_{3}$ tends to yield one order magnitude larger than the magnitude of $f$ and at a smaller axial position for smaller values of $W_{2}$.

\subsection{Optimal design observations}

From the numerical simulations, three relevant geometric parameters were identified for the 3D flow-focusing junction: the distance between the inner inlet channel and the outlet channel, $W_{2}$, the width of the outlet channel, $W_{3}$, and its length $L_{3}$ (see Fig. 1). As stated before, to achieve the
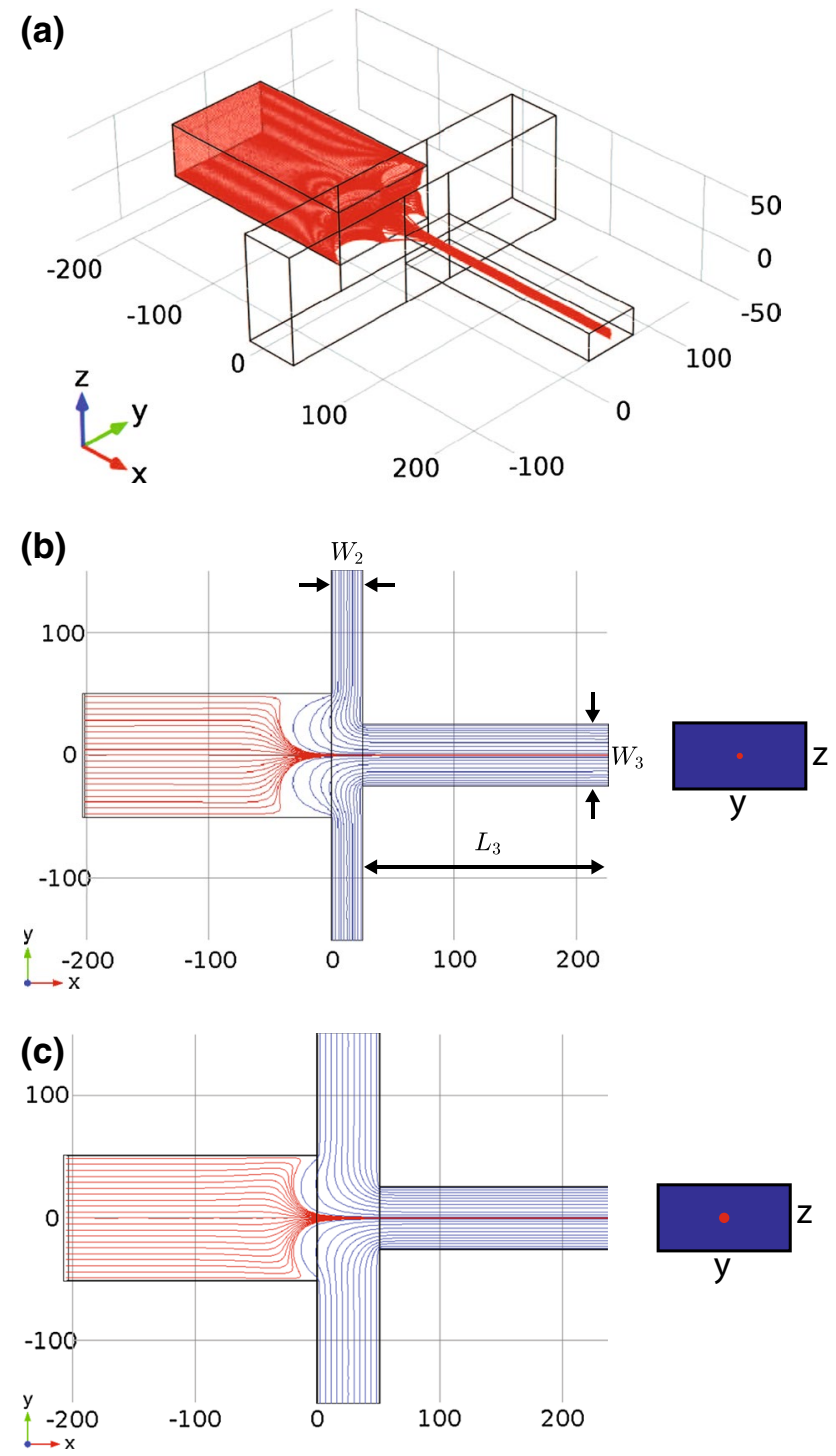

Fig. 2 Streamline images showing the confinement of the inner fluid by the outer fluid. a The 3D structure of the interface between the inner and outer fluid. b,c Streamlines of the inner fluid (red) and outer fluid (blue) along the symmetry plane, $z=0$. The cross sections located at the outlet show the $3 \mathrm{D}$ confinement of the jet (jet radii are not to scale). $\mathbf{b} W_{2}=25, W_{3}=50, \mathbf{a}, \mathbf{c} W_{2}=50, W_{3}=50$. Flow is in the positive $\mathrm{x}$-direction (color figure online)

production of small droplets, the magnitude of $f$ should be as large as possible, while the asymptotic values of $u_{x}$ and $f$ should be reached as close to the junction as possible in order to have shorter breakup lengths. Our results show that this is best achieved by minimizing both the outlet channel width $W_{3}$ and the inlet channel width $W_{2}$. Channels with a width $W_{3}=50 \mu \mathrm{m}$ were chosen for all our experiments in order to reduce the probability of clogging the microchannels. The length of the outlet channel should be just long enough for these values to actually reach their asymptotic values (see Fig. 3) without increasing unnecessarily the 

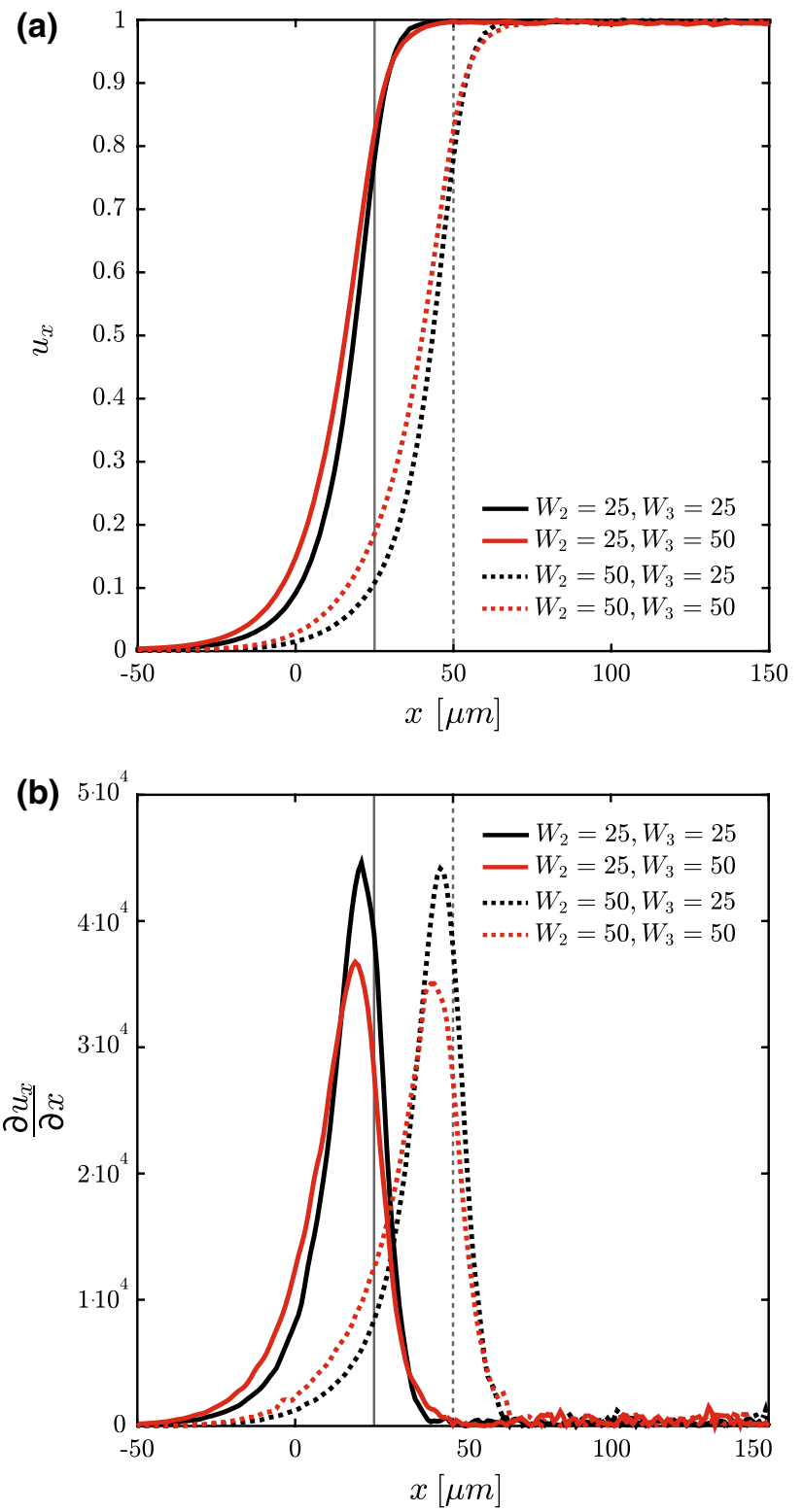

Fig. 3 Downstream evolution of the dimensionless computed velocity $u_{x}$ along the center axis (a) and its derivative with respect to the streamwise direction (b), for two values of $W_{2}$ and $W_{3}$. The results show a strong dependence on $W_{2}$, while $W_{3}$ shows only a minor impact. The solid and dashed gray lines indicate the position of the entrance of the outlet channel for the two values of $W_{2}, 25$ and $50 \mu \mathrm{m}$, respectively

pressure drop. To this purpose, channels with two different lengths $L_{3}: 125$ and $250 \mu \mathrm{m}$ were chosen.

Generally, drops produced in the dripping regime are highly monodisperse, with low production frequency and with diameter of the same order as the outlet channel width (Castro-Hernández et al. 2009). Droplets generated in the jetting regime have a higher production frequency with a size comparable to that of the jet.

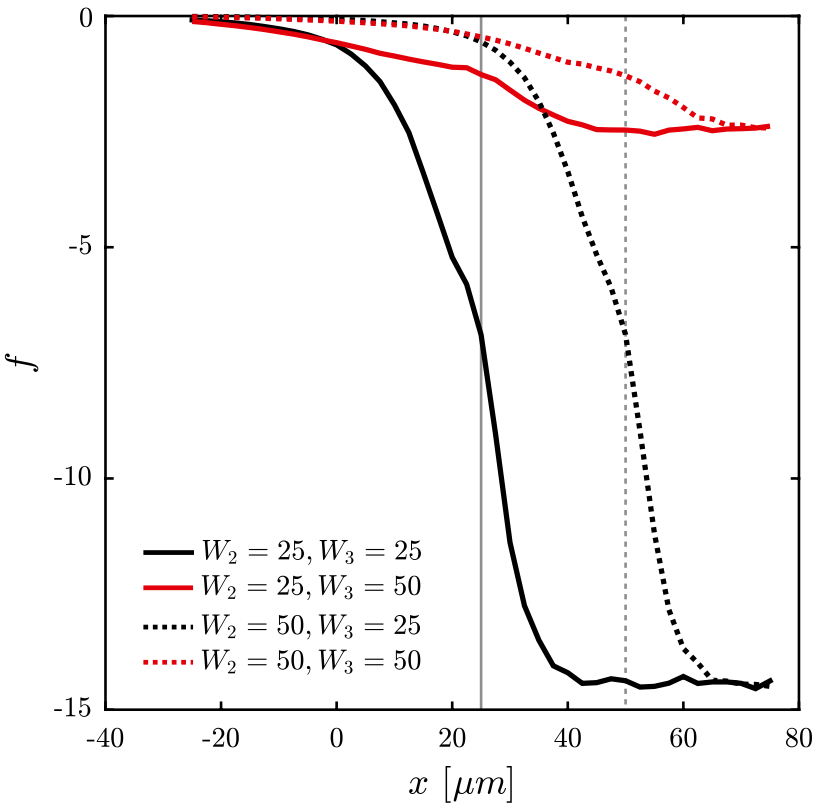

Fig. 4 Downstream evolution of the dimensionless computed orthogonal shear stress gradient $f$ along the center axis. The asymptotic value of $f$ is shown to be determined by $W_{3}$, while $W_{2}$ strongly influences the position at which this value is obtained. The solid and dashed gray lines indicate the position of the entrance of the outlet channel for the two values of $W_{2}, 25$ and $50 \mu \mathrm{m}$, respectively

Two different jetting regimes have been reported: narrowing and widening, where these names reflect the shape adopted by the jet along the flow direction inside the channel (Utada et al. 2007; Castro-Hernández et al. 2009). Narrowing jets are formed when, due to the outer stream, the viscous stresses on the interface overcome surface tension confinement forces. In these situations, the outer velocity is larger than the inner velocity, and as a consequence, the jet stretches. Working under the narrowing regime leads to the production of smaller monodisperse droplets from the same device when operated under the appropriate conditions. It has been reported (Marín et al. 2009) that the narrowing regime occurs when the outer Reynolds number is $R e_{o}=\rho_{o} U_{o} W_{3} / \mu_{o} \ll 1$ and the outer capillary number $C a_{o}=\mu_{o} U_{o} / \sigma$ is larger than a certain value of order unity (Gordillo et al. 2014). To observe a slender cone-jet transition the inner-to-outer velocity ratio needs to satisfy $U_{i} / U_{o} \ll 1$. Since PDMS is an elastic material, the accurate determination of these parameters is not possible for our experiments, although the conditions must be satisfied due to the small dimension of the outlet channel $\left(W_{3}=50 \mu \mathrm{m}\right)$ and the high viscosity value of the outer fluid $\left(\mu_{o}=1000 \mathrm{cP}\right)$.

In our experiments, droplets with a diameter $D_{d}=22 \mu \mathrm{m}$ ( $P_{o}=1700 \mathrm{mbar}, P_{i}=1637 \mathrm{mbar}$ ), have an experimentally measured droplet velocity $U_{d}=0.05 \mathrm{~m} / \mathrm{s}$ giving $R e_{d}=\rho_{o} U_{d} W_{3} / \mu_{o} \simeq 10^{-3}$ and $C a_{d}=\mu_{o} U_{d} / \sigma \simeq 6$. 

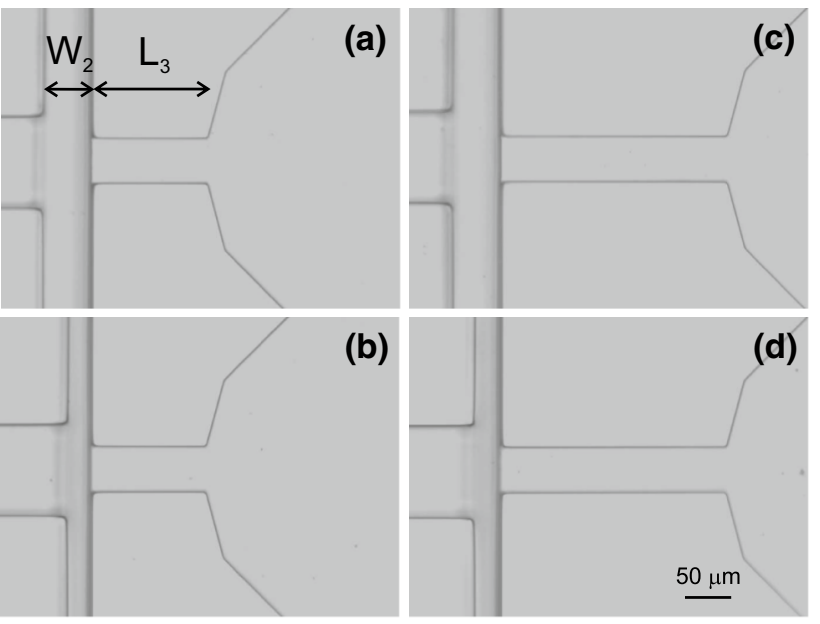

Fig. 5 Images depicting the four different tested designs: a Design 1: $L_{3}=125 \mu \mathrm{m}, W_{2}=50 \mu \mathrm{m}$; b Design 2: $L_{3}=125 \mu \mathrm{m}, W_{2}=25 \mu \mathrm{m}$; c Design 3: $L_{3}=250 \mu \mathrm{m}, W_{2}=50 \mu \mathrm{m}$; d Design 4: $L_{3}=250 \mu \mathrm{m}$, $W_{2}=25 \mu \mathrm{m}$

Table 1 Dimensions for the different fabricated designs

\begin{tabular}{lllll}
\hline Design & $\# 1$ & $\# 2$ & $\# 3$ & $\# 4$ \\
\hline$L_{3}$ & 125 & 125 & 250 & 250 \\
$W_{2}$ & 50 & 25 & 50 & 25 \\
$W_{1}$ & 100 & & & \\
$H_{1}$ & 50 & & & \\
$H_{2}$ & 100 & & & \\
$W_{3}$ & 50 & & & \\
$H_{3}$ & 25 & & \\
\hline
\end{tabular}

All units are in $[\mu \mathrm{m}]$

Since we do not observe droplet coalescence, they move with the same velocity as the outer fluid, and this means that the conditions for having a narrowing jet are fulfilled.

Taking all this information into consideration, four designs were chosen to be tested. Table 1 lists all the dimensions of the different designs. Figure 5 shows images of the design taken under the microscope.

Above a certain threshold of $\mathrm{Ca}_{o}$, increasing the outer capillary number, while keeping all other parameters constant, has the effect of larger breakup length of the jet and higher production frequency, as reported in the literature for the two-dimensional case (Cubaud and Mason 2008) and axisymmetric case (Castro-Hernández et al. 2012). Figure 6 shows that, indeed, for all the pressure values tested with designs 1 and 2 , the jet breaks up outside the outlet channel and that the generated droplets are polydisperse (polydispersity index PDI $>60 \%$ ).

Figure 7a and c shows that for designs 3 and 4, it was possible to obtain, quite easily, droplets with a diameter

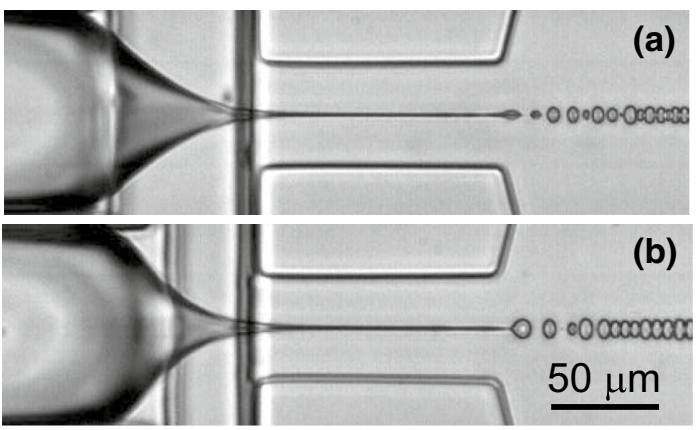

Fig. 6 The jet does not break inside the channel for all the pressure values tested with designs 1 and 2. a Design 1: $P_{o}=1100 \mathrm{mbar}$, $P_{i}=1062 \mathrm{mbar}, \quad D_{d}=14 \mu \mathrm{m} ; \quad$ b Design 2: $P_{o}=1100 \mathrm{mbar}$, $P_{i}=1021 \mathrm{mbar}, D_{d}=17 \mu \mathrm{m}$

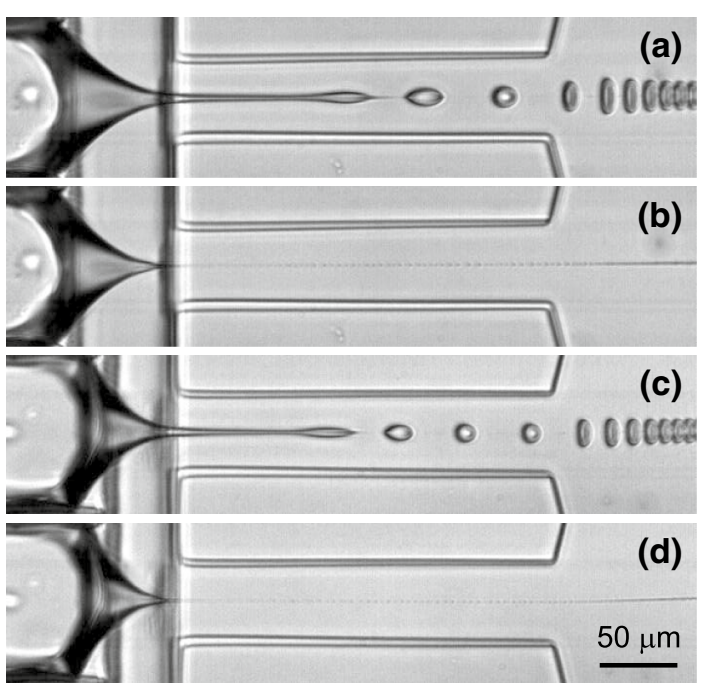

Fig. 7 Although it is possible to obtain small droplets with both designs 3 and 4 , the jet is more stable for the latter. a Design 3, $P_{o}=1100 \mathrm{mbar}, \quad P_{i}=1070 \mathrm{mbar}, \quad D_{d}=12 \mu \mathrm{m} ; \quad$ b Design 3 , $P_{o}=1100 \mathrm{mbar}, \quad P_{i}=1065 \mathrm{mbar}, \quad D_{d}=2 \mu \mathrm{m} ; \quad$ c Design 4 , $P_{o}=1100 \mathrm{mbar}, \quad P_{i}=1034 \mathrm{mbar}, \quad D_{d}=13 \mu \mathrm{m} ; \quad$ d Design 4 , $P_{o}=1100 \mathrm{mbar}, P_{i}=1031 \mathrm{mbar}, D_{d}=1.6 \mu \mathrm{m}$

$D_{d} \sim 10 \mu \mathrm{m}$ and a polydispersity index PDI $<1 \%$. An optimized minimum inner-to-outer pressure ratio needs to be found empirically for each design to obtain the desired droplet size. As depicted in Figure $7 \mathrm{~b}$ and d, designs 3 and 4 also allow for the production of micron-sized droplets when operated under the appropriate conditions. Nevertheless, design 4 was found to produce more stable jets when undesirable perturbations from the surrounding, such as floor vibrations, occurred.

It is possible that deformation of the device, as it is made of PDMS, may disturb jet expansion and induce retraction into the inlet chamber. The pressure drop in PDMS channels, with the device working at a pressure of $45 \mathrm{kPa}$, was reported to be up to $35 \%$ less than in a 
rigid-walled channel, and the effect is expected to decrease proportionally with the channel wall thickness (Hardy et al. 2009). For future experiments, the use of glass or other stiffer materials for the fabrication of the microfluidic 3D flow-focusing junction will be required. Nevertheless, rapid prototyping with PDMS allowed for a faster experimental verification of the computed values and at the test conditions. The jet diameter is slightly thinner in design 4 , which agrees with the experimental observation as depicted in Fig. 7 and as predicted in the simulations, which do show a stronger decrease of approximately $30 \%$ for design 4, see Fig. 2.

In the classical planar flow-focusing devices, the length of the constriction is of the same order as the channel width. The dispersed phase is then blocked within the constriction, and the pressure upstream increases leading to the squeezing of the jet. In our designs, the constriction is much longer than the channel width, allowing the hydrodynamic generation of a long jet that eventually breaks up into droplets much smaller than the constriction, reducing clogging and having no wettability problems. Our fabrication approach is also cheaper than the current processes since only one photolithographic mask is used for the SU-8 molds fabrication of identical PDMS slabs. In the future, other materials, such as stainless steel, could be used with similar dimensions. All these features, in addition to a reduced chance of clogging, are desirable for the present trend in emulsification techniques, where the passage of larger particles, liquid concentrates used as feeds, and reduced maintenance are required (Schroën et al. 2015). Based on our observations, the optimal geometry corresponds to that of design 4 .

\section{Conclusions}

In this manuscript, we present a numerical and experimental study on the geometry of a truly 3D microfluidic flowfocusing device for the generation of micrometer-sized droplets. The main advantage of using such a 3D device is that the axisymmetric jet is properly centered at the outlet channel that eventually breaks up into droplets. As a consequence, the wetting properties of the material are no longer relevant, avoiding the use of chemicals to control the wetting behavior of the microchannels walls.

Three relevant geometric parameters were identified: the distance between the inner inlet channel and the outlet channel, the width of the outlet channel, and its length. With the data obtained from finite element simulations, four different designs were selected and fabricated using soft lithography techniques. Under the adequate operating conditions, it was possible to obtain droplets of $\sim 1 \mu \mathrm{m}$ in diameter from a channel of $50 \mu \mathrm{m}$ in width. The optimal configuration studied corresponds to that of design 4: $L_{3}=250 \mu \mathrm{m}, W_{2}=25 \mu \mathrm{m}, W_{1}=100 \mu \mathrm{m}, H_{1}=50 \mu \mathrm{m}$, $H_{2}=100 \mu \mathrm{m}, W_{3}=50 \mu \mathrm{m}$ and $H_{3}=25 \mu \mathrm{m}$.

Acknowledgments The fabrication and design of the microfluidic devices were possible with the advice and assistance of S. Schlautman and H.S. Rho. The authors are grateful to G. Lajoinie for his valuable assistance during the setup preparation. We also would like to acknowledge the useful comments of Prof. J. M. Gordillo during the design phase. This work has been partially financed with a grant from the V Plan Propio de Investigación of the University of Seville, Project TEP-5984 from Consejería de Economía, Innovación, Ciencia y Empleo and NanoNextNL, a micro- and nanotechnology consortium of the Government of the Netherlands and 130 partners.

\section{References}

Anderson JR, Chiu DT, Wu H, Schueller OJ, Whitesides GM (2000) Fabrication of microfluidic systems in poly (dimethylsiloxane). Electrophoresis 21:27-40

Anna SL, Bontoux N, Stone HA (2003) Formation of dispersions using flow focusing in microchannels. Appl Phys Lett 82:364-366

Carlier J, Arscott S, Thomy V, Fourrier JC, Caron F, Camart JC, Druon C, Tabourier P (2004) Integrated microfluidics based on multi-layered SU-8 for mass spectrometry analysis. Institute of Physics Publishing, Bristol

Castro-Hernández E, Gundabala V, Fernández-Nieves A, Gordillo JM (2009) Scaling the drop size in coflow experiments. New J Phys 11(075):021

Castro-Hernández E, Campo-Cortés F, Gordillo JM (2012) Slenderbody theory for the generation of micrometre-sized emulsions through tip streaming. J Fluid Mech 698:423-445

Chiu YJ, Cho SH, Mei Z, Lien V, Wu TF, Lo YH (2013) Universally applicable three-dimensional hydrodynamic microfluidic flow focusing. Lab Chip 13:1803-1809

Cubaud T, Mason TG (2008) Capillary threads and viscous droplets in square microchannels. Phys Fluids 20(5):053,302

Dhanaliwala AH, Chen JL, Wang S, Hossack JA (2012) Liquid flooded flow-focusing microfluidic device for in situ generation of monodisperse microbubbles. Microfluid Nanofluid 14(3-4):457-467

Engl W, Backov R, Panizza P (2008) Controlled production of emulsions and particles by milli- and microfluidic techniques. Curr Opin Colloid Interface Sci 13(4):206-216

Frankowski M, Theisen J, Kummrow A, Simon P, Ragusch H, Bock N, Schmidt M, Neukammer J (2013) Microflow cytometers with integrated hydrodynamic focusing. Sensors 13(4):4674-4693

Gañán-Calvo AM (1998) Generation of steady liquid microthreads and micron-sized monodisperse sprays in gas streams. Phys Rev Lett 80:285-288

Garstecki P, Fuerstman MJ, Whitesides GM (2005) Nonlinear dynamics of a flow-focusing bubble generator: an inverted dripping faucet. Phys Rev Lett 94(23):234,502

Gijsbertsen-Abrahamse A (2004) Status of cross-flow membrane emulsification and outlook for industrial application. J Membr Sci 230(1-2):149-159

Golden JP, Kim JS, Erickson JS, Hilliard LR, Howell PB, Anderson GP, Nasir M, Ligler FS (2009) Multi-wavelength microflow cytometer using groove-generated sheath flow. Lab Chip 9(13):1942-1950 
Gordillo JM, Sevilla A, Campo-Cortés F (2014) Global stability of stretched jets: conditions for the generation of monodisperse micro-emulsions using coflows. J Fluid Mech 738:335-336

Hardy BS, Uechi K, Zhen J, Pirouz Kavehpour H (2009) The deformation of flexible PDMS microchannels under a pressure driven flow. Lab Chip 9(7):935

Lee W, Walker LM, Anna SL (2009) Role of geometry and fluid properties in droplet and thread formation processes in planar flow focusing. Phys Fluids 21(3):032,103

Marín AG, Campo-Cortés F, Gordillo JM (2009) Generation of micron-sized drops and bubbles through viscous coflows. Colloids Surf A Physicochem Eng Asp 344:2-7

Nisisako T, Torii T (2008) Microfluidic large-scale integration on a chip for mass production of monodisperse droplets and particles. Lab Chip 8(2):287

Paiè P, Bragheri F, Vazquez RM, Osellame R (2014) Straightforward 3D hydrodynamic focusing in femtosecond laser fabricated microfluidic channels. Lab Chip 14(11):1826

Rosenauer M, Buchegger W, Finoulst I, Verhaert P, Vellekoop M (2011) Miniaturized flow cytometer with $3 d$ hydrodynamic particle focusing and integrated optical elements applying silicon photodiodes. Microfluid Nanofluid 10(4):761-771

Schroën K, Bliznyuk O, Muijlwijk K, Sahin S, Berton-Carabin CC (2015) Microfluidic emulsification devices: from micrometer insights to large-scale food emulsion production. Curr Opin Food Sci 3:33-40
Scott R, Sethu P, Harnett CK (2008) Three-dimensional hydrodynamic focusing in a microfluidic coulter counter. Rev Sci Instrum 79(4):046,104

Sim SPC, Kang TG, Yobas L, Holtze C, Weitz DA (2010) The shape of a step structure as a design aspect to control droplet generation in microfluidics. J Micromech Microeng 20(3):035,010

Simonnet C, Groisman A (2005) Two-dimensional hydrodynamic focusing in a simple microfluidic device. Appl Phys Lett 87(11): 114,104

Sugiura S, Nakajima M, Iwamoto S, Seki M (2001) Interfacial tension driven monodispersed droplet formation from microfabricated channel array. Langmuir 17(18):5562-5566

Taylor GI (1964) Conical free surfaces and fluid interfaces. In: Proceedings of the 11th international congress of applied mechanics (Munich), pp 790-796

Utada AS, Lorenceau E, Link DR, Kaplan PD, Stone HA, Weitz D (2005) Monodisperse double emulsions generated from a microcapillary device. Science 308:537-541

Utada AS, Fernández-Nieves A, Stone HA, Weitz D (2007) Dripping to jetting transitions in co-flowing liquid streams. Phys Rev Lett 99(094):502

Zhuang G, Jensen TG, Kutter JP (2012) Detection of unlabeled particles in the low micrometer size range using light scattering and hydrodynamic $3 \mathrm{~d}$ focusing in a microfluidic system. Electrophoresis 33(12):1715-1722 